\title{
Wassermilben (Acari: Hydrovolziidae et Hydrachnellae) aus Algerien*
}

\author{
Carl Bader \\ Naturhistorisches Museum, Acarologische Abteilung, Augustinergasse 2, CH-4001, Basel, Schweiz
}

Keywords: Acari (Hydrachnellae), Algeria, Groundwater

\begin{abstract}
In the material collected from wells, hyporheic waters, and springs in western Algeria seven species of watermites have been found. Three of these are new to science: Acherontacarus tuberculatus nov. spec., Neumania (s. str.) algeriensis nov. spec., and the representative of a new genus Neoacherontacarus dividuus nov. gen., nov. spec. The male of Limnesia (s. str.) granulosa Walter, 1931 is discovered and for the first time described.
\end{abstract}

\section{Résumé}

Sept espèces d'Hydracariens ont été trouvées dans le matériel collecté en Algérie de l'ouest dans des puits, des eaux hyporhéiques et des sources. Trois de celles-ci sont nouvelles: Acherontacarus iuberculatus nov. spec., Neumania (s. str.) algeriensis nov. spec., et Neoacherontacarus dividuus nov. gen., nov. spec., Le mâle auparavant inconnu de Limnesia (s. str.) granulosa Walter, 1931 est pour la première fois décrit.

\section{Einleitung}

Die Bio-Speleologische Werkgruppe von 'Speleo Nederland' stellte sich, nach einer ersten Abklärung im Jahre 1978, die Aufgabe, im westlichen Teil Algeriens die Grundwasserfauna zu erforschen. Im Jahre 1981 wurden mit einigem Erfolg die Grundwasserbiotope Algeriens untersucht. Zwei Jahre später, also 1983, beschäftigten sich 7 Mitglieder der Gruppe intensiv mit der Grundwasserfauna Al- geriens. In der im Jahre 1986 publizierten Zusammenfassung 'Survey of the Groundwater-Stations investigated in 1981 and 1983' (Notenboom \& De Winter, 1986) wurden die technischen Daten der zahlreichen Fundstellen festgehalten. Drei Biotope standen im Vordergrund der Erhebungen. Diese lieferten nur ein bescheidenes Material an Wassermilben.

1. Sodbrunnen (= wells): Mit Hilfe eines Cvetkov-Schliessnetzes (Durchmesser $30 \mathrm{~cm}$ ) wurden die Tiere im Wasser aufgestört und gefangen. In den uns zur Verfügung gestellten positiven Proben fanden sich meist vereinzelte, gelegentlich auch viele Wassermilben. Sie waren begleitet von einigen wenigen nicht bestimmbaren terrestrischen Acarinen der Unterordungen Prostigmata, Mesostigmata und Cryptostigmata. Diese gerieten beim Sammeln zufälligerweise in die Proben. Die in den Sodbrunnen gefundenen Hydracarinen Hydryphantes algeriensis, Limnesia granulosa, Neumania elliptica und $N$. algeriensis sind typische Oberflächenformen.

2. Interstitial: Die Grabungen mit der KaramanChappuis-Methode neben den Fliessgewässern bis in die Nähe des Meeresufers sollten aus dem hyporheischen Bereich die echten stygobionten Hydracarinen liefern. Prinzipiell sind diese farblos, blind und klein (unter $0,5 \mathrm{~mm}$ ). Da sie sich kaum bewegen, dürften sie nicht aufgefallen sein, sie fehlen im vorliegenden Material. Die verwendeten

* Dutch biological and speleological explorations in Algeria, no. 7. 
Netze mit einer Maschenweite von $400 \mu \mathrm{m}$ liessen diese Tiere passieren! Die Acherontacariden sind bis jetzt als Bewohner des Interstitials bezeichnet worden. Zehn Exemplare von Acherontacarus tuberculatus nov. spec. fanden sich indessen in einer offenen 'gefassten Quelle'. Sie sind grösser als $0,4 \mathrm{~mm}$ und konnten daher bei der Verwendung der oben erwähnten Netze entdeckt werden. Sie besitzen wie die echten Stygobionten keine Augen, sind jedoch im Gegensatz zu den farblosen Stygobionten deutlich braun gefärbt. $A$. tuberculatus lässt sich vorerst nicht als echte Stygobionte einstufen. Dieselbe Bemerkung gilt auch für Neoacherontacarus dividuus nov. spec.

3. Quellen, Karstquellen etc.: Die uns zur Verfügung gestellten Daten sind ungenügend. Die Unterscheidung in Rheo-, Limno- und Helokrenen wurde nicht vorgenommen. Angaben über Moosbewuchs fehlen. Als echte Quellbewohner sind hier Panisus clypeolatus und Nilotonia robusta zu nennen.

Die sonst üblichen Studien mit korrekt konserviertem Material konnten hier nur teilweise angewendet werden. Die folgenden Diagnosen müssen in Hinsicht auf die Feinstrukturen, insbesondere der Haut, unvollständig, bleiben. Einwandfreie, der Wissenschaft dienliche mikroskopische Präparate liessen sich nicht herstellen, doch dürften die im Naturhistorischen Museum Basel aufbewahrten Präparate für eine Identifizierung genügen.

Die Herren Dr. L. Botosaneanu und Ir. J. Notenboom hatten die Freundlichkeit, mein Manuskript vor der Drucklegung zu überprüfen, um einige sich aufdrängende Ergänzungen vorzunehmen. Ihnen gehört mein bester Dank für das gute Gelingen dieser Publikation.

\section{Systematische Teil}

\section{HYDROVOLZIIDAE Thor, 1905}

\section{Acherontacarus Viets, 1932}

Die hier beschriebene neue Art Acherontacarus tuberculatus veranlasst uns, die in einer früheren Publikation gemachten Angaben (Bader, 1983) über Acherontacarus Viets, 1932 zu präzisieren.
Die damals aufgestellten drei Subgenera müssen vorerst bestehen bleiben. Die von Cook (1967) eingeführte $A$. (Acherontacaropsis) bleibt weiterhin suspekt. Nach Cook soll die zu diesem Taxon rechnende $A$. cedro Lundblad, 1961 deutliche Augenkapseln besitzen. Lundblad (1962) erwähnt diese nicht, aus seiner in Tafel $1 / 1$ publizierten Photo verbleibt die Existenz von Augenkapseln unklar. Die beiden anderen, augenlosen Subgenera $A$. (Acherontacarellus) und $A$. (sensu strictu) sind berechtigt. Die folgende Bestimmungstabelle gibt den gegenwärtigen Stand der bisher nur im mediterranen Gebiet (inklusive Kanarischen Inseln) nachgewiesenen Gattung.

1. Augenkapseln vorhanden (Cook: but reduced).

Oberflächenform. Subgenus Acherontacaropsis

Einzige Art: cedro Lundblad, 1962 (Kanarische Inseln)

- Augenkapseln fehlen. Interstitielle Formen ........ 2

2. Mediannaht der 1. Epimeren nur angedeutet, breitovale Formen. Subgenus Acherontacarus

Zwei Arten: halacaroides Viets, 1932 (Jugoslawien)

fonticolus Viets, 1934. Nymphe (Jugoslawien)

- Mediannaht der 1. Epimeren deutlich ausgebildet, längsovale

Formen. Subgenus Acherontacarellus (siehe Abb. 1) ... 3

3. $\mathrm{dl}_{2}$ und $\mathrm{dl}_{3}$ grösser als die dazugehörenden $\operatorname{lgl}_{2}$ und $\operatorname{lgl} \mathrm{gl}_{3}$ vietsi Angelier, 1951 (Korsika)

$-\mathrm{dl}_{2}$ und $\mathrm{dl}_{3}$ kleiner als $\lg \mathrm{l}_{2}$ resp. $\operatorname{lgl}_{3} \ldots \ldots \ldots \ldots \ldots .4$

4. $\operatorname{lgl}$ doppelt so lang wie breit bicornis Cook, 1974 (Spanien)

$-\operatorname{lgl}_{1}$ gedrungen, gleich lang wie breit $\ldots \ldots \ldots \ldots \ldots \ldots$

5. Genitale 1 breiter als lang rutilans Angelier, 1951 (Korsika)

- Genitale 2 länger als breit. Seitenrand des Post-Dorsale mit Tuberkeln besetzt ...................6 6

6. Am $P_{2}$ stehen ventral 2 feine Haare cicolanii Bader, 1983 (Sardinien)

- Am $P_{2}$ sind keine Haare inseriert tuberculatus nov. spec. (Algerien)

\section{Acherontacarus (Acherontarellus) tuberculatus} nov. spec. (Abb. 1 und 2)

Fundorte: Sta. 83-76 (28.4.1983), Wilaya Saïda, gefasste Quelle, $4 \mathrm{~km}$ östlich von Saïda, $34^{\circ} 49^{\prime} \mathrm{N} 00^{\circ} 11^{\prime} 0$, Höhe: $1000 \mathrm{~m}$. Temperatur: $14,2^{\circ} \mathrm{C} .7$ Weibchen (1 Holotypus), 1 Männchen, 2 Nymphen.

Sta. 83-32 (18.4.1983), Wilaya Tlemcen, KaramanChappuis-Grabung am Strand von Sidna Youcha, 7 km OstNordost von Ghazaouet, $37^{\circ} 7^{\prime} \mathrm{N} 1^{\circ} 46^{\prime} \mathrm{W}$, Temperatur: $18,2^{\circ} \mathrm{C}$. 2 Weibchen. 

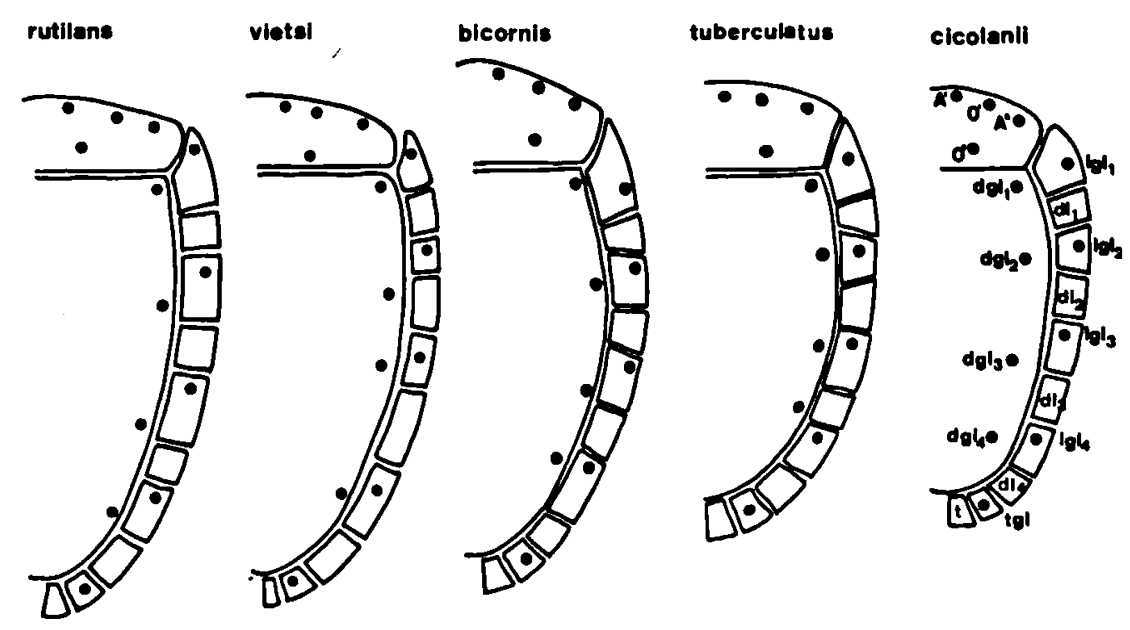

$800 \mathrm{u}$

Abb. 1. Acherontacarus Subgenus Acherontacarellus. Schemazeichnung der Dorsalfläche der fünf zur Zeit bekannten Arten.

Weibchen

A. tuberculatus steht $A$. cicolanii sehr nahe. Im Folgenden werden die zur Verfügung stehenden Messdaten der Vergleichsart in Klammern gesetzt.

Dorsum: Körpergrösse 860/550 (860/610). PraeDorsale 168/343 (150/330) mit den 4 Haarpaaren, den Antenniformia und Ocularia der Hydrachnellae gleichgesetzt. Post-Dorsale 624/406 (590/370), am Rande stehen zahlreiche deutlich erkennbare Tuberkeln (tuberculatus!). Die $4 \mathrm{Haare}^{\mathrm{dgl}_{1-4}}$ sind nach der Hydrachnellen-Nomenklatur benannt, die Drüsen fehlen. Die Bezeichnungen der 10 randständigen kleinen Schilder sind in der Publikation von Bader (1983) eingeführt worden (siehe Abb. 1).

Ventrum: Körpergrösse $880 / 550$. Die mediane Trennungslinie der aneinander stossenden 1. Epimeren ist sehr prägnant. Genitalorgan 150/70 (175/80). Die Genitale 1-3 sind gleich angeordnet wie bei der Vergleichsart. Das Exkretale misst 200/200 (225/190), das Post-Exkretale 170/280 (170/260). Im Innern des Körpers vom Holotypus liegt ein grosses Ei mit 310 Durchmesser.

Gnathosoma: Infracapitulum 205/175 (225/ 160). Palpen: $43-142-96-111-40=432$ $(37-144-80-90-37=388)$. Cheliceren $263(250)$. Die Behaarung der Palpen ist bei tuberculatus noch spärlicher als bei cicolanii. Bei der letzteren stehen an der Ventralseite von $P_{2}$ distalwärts 2 feine
Haare, die bei der neuen Art fehlen.

Extremitäten:

$\begin{array}{llllrllll}\text { I } & 28 & 144 & 93 & 96 & 102 & 109 & = & 572 \\ \text { II } & 31 & 179 & 93 & 96 & 105 & 123 & = & 637 \\ \text { III } & 34 & 151 & 99 & 102 & 109 & 144 & = & 639 \\ \text { IV } & 31 & 155 & 99 & 114 & 114 & 144 & = & 657\end{array}$

Zum Vergleich folgen die Messdaten von $A$. cicolanii:

$\begin{array}{lllllllll}\text { I } & 31 & 207 & 96 & 117 & 127 & 158 & = & 736 \\ \text { II } & 43 & 232 & 96 & 114 & 136 & 179 & = & 800 \\ \text { III } & 34 & 210 & 93 & 111 & 127 & 170 & = & 745 \\ \text { IV } & 34 & 182 & 93 & 127 & 155 & 192 & = & 783\end{array}$

Wenn auch die bestehende Variabilität der Messdaten innerhalb einer Art, sie dürfte bei 12\% liegen, berücksichtigt wird, so sind die Werte der beiden Arten niemals als übereinstimmend zu bewerten. Die Beinmasse dienen hier entscheidend zur Trennung der beiden Arten! Die Totalmasse der vier Beine bewegen sich in einem ungewohnt engen Rahmen: 570-657 resp. 736-800. Auffallend ist das 2 . Beinglied, in ihm stecken die maximalen Beinglieder: 179 resp. 232! Auf Grund einer grossen Zahl vorliegender Messdaten vieler Wassermilben-Gattungen kann (vorläufig) festgestellt werden, dass Acherontacarus betr. der Gliedlängen eine Ausnahmestellung einnimmt! 

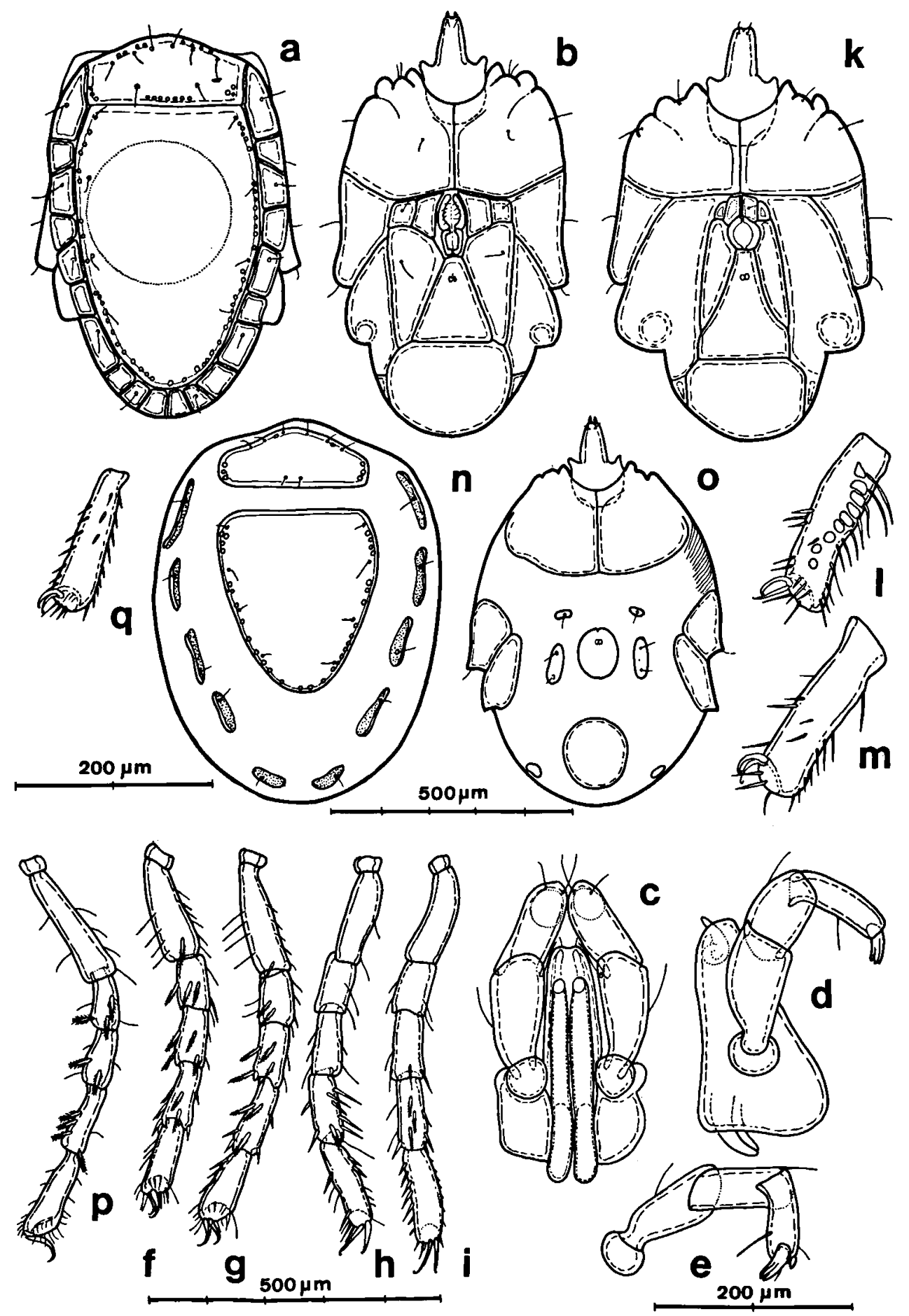

Abb. 2. Acherontacarus (Acherontacarellus) tuberculatus nov. spec., Weibchen: a, dorsal; b, ventral; c, Gnathosoma dorsal; d, Gnathosoma lateral; e, Palpe innen; f-i, Beine I-IV. Männchen: k, ventral; l, Bein IV, 6. Glied innen; m, Bein IV, 6. Glied aussen. A. (Acherontacarellus) cicolanii Bader, 1983, Weibchen: p, Bein I; q, Bein IV, 6. Glied aussen. 
Über die Behaarung der 4 Beine geben Abb. $2 \mathrm{f}-\mathrm{i}$ Auskunft, der Vergleich des 1. Beines von $A$. cicolanii (Abb. 2p) lässt eine gewisse Übereinstimmung erahnen.

\section{Männchen}

Dorsum: Körpergrösse 880/645. Prae-Dorsale 137/393, Post-Dorsale 624/456.

Ventrum: Körpergrösse 880/645. Genitalorgan 69/72. Die Genitale 1 und 2 sind gegenüber dem Weibchen anders angeordnet, das Genitale 3 misst 262/69. Exkretale 275/225, Post-Exkretale 175/ 306.

Gnathosoma: Infracapitulum 256/168.

Nymphe

Dorsum: Körpergrösse 820/600. Prae-Dorsale 119/324. Post-Dorsale 380/337.

Ventrum: Körpergrösse 770/600. Vorderes Epimeralgebiet 250/418. Exkretale 81/106, PostExkretale 150/144.

\section{Neoacherontacarus nov. gen.}

Die Ventralansicht in Abb. 3a bestätigt die nahe Verwandtschaft mit Acherontacarus. Dorsal ergibt sich jedoch eine entscheidende Differenz: Am PostDorsale sondert sich hinten ein kleines Schild ab. Eine solche Absonderung zeigt sich nur noch bei einigen Nymphen der Hydrovolziidae.

Neoacherontacarus dividuus nov. spec. (Abb. 3)

Fundort: Sta. 83-26 (17.4.1983), Wilaya Tlemcen, Wasserfälle bei El-Ourit, $5 \mathrm{~km}$ Ost-Südost von Tlemcen, 'small pool fed by

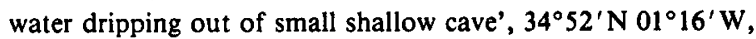
Höhe: 980 m. 1 Männchen (Holotypus), 1 Nymphe.

\section{Männchen}

Dorsum: Körpergrösse 920/820. Mit Ausnahme des Post-Post-Dorsale stimmt der Aufbau mit Acherontacarus überein. Das Prae-Dorsale misst 137/447, die auf ihm inserierten Haare sind nicht abweichend angeordnet. Das eigentliche PostDorsale ist 609 lang und 560 breit. In ihm finden sich nur 3 der dgl-Haare. Das anschliessende PostDorsale misst 124/323, auf ihm steht das $\mathrm{dgl}_{4}$. Die Seitenschilder sind laut Schema in Abbildung 1 an- geordnet. Es fällt auf, dass $\lg 1_{4}$ auffallend lang ist. Ventrum: Körpergrösse 1020/820. Die beiden vorderen Epimeren stossen in einer deutlichen Naht zusammen. Das Genitalorgen ist 100 lang und 106 breit, ihm vorgelagert ist das relativ grosse Genitale 1, die Nummer 2 ist bedeutend kleiner. Nummer 3 misst 324/125, das Exkretale 287/343, das PostExkretale 218/325.

Gnathosoma: Infracapitulum 250/206. Palpen: 46-158-90-117-53 = 464. Cheliceren 280. Behaarung siehe Abb. $3 \mathrm{c}-\mathrm{e}$.

\begin{tabular}{llllllllr}
\multicolumn{2}{l}{ Extremitäten: } & & & & & \\
I & 69 & 225 & 144 & 156 & 162 & 162 & $=$ & 918 \\
II & 87 & 275 & 150 & 162 & 187 & 200 & $=$ & 1061 \\
III & 87 & 349 & 131 & 193 & 200 & 262 & $=$ & 1222 \\
IV & 87 & 343 & 144 & 256 & 231 & 225 & $=$ & 1286
\end{tabular}

Das längste Beinglied findet sich in dieser neuen Gattung resp. Art wie bei Acherontacarus in der Reihe der zweiten Beinglieder. Es fällt auf, dass die ersten und dritten Glieder der vier Beine ungefähr gleich lang sind. Bei den Totallängen ergibt sich von 918 auf 1286 eine deutliche Steigerung. Die Einzelheiten der Behaarung können aus Abbildung $3 \mathrm{f}-\mathrm{i}$ ersehen werden, hier fällt auf, dass die gefiederten Haare nur auf den ersten zwei Beinen zu finden sind.

\section{Nymphe}

Die im gleichen Fundort entdeckte Nymphe kann der neuen Art zugewiesen werden, das Post-PostDorsale des Männchens fehlt.

Dorsum: Körpergrösse 745/620. Prae-Dorsale 144/393 mit je 4 Haaren. Post-Dorsale 430/418. Im Seitenrand liegen je 5 Platten, sie sind alle mit einem Haar ausgerüstet, entsprechen daher laut Schemazeichnung in Abb. 1 den $\mathrm{lgl}_{1-4}$ resp. tgl.

Ventrum: Körpergrösse 820/620. Die beiden vorderen Epimerengruppen verschmelzen median, eine ausgeprägte Naht ist nicht vorhanden. Die Genitale 1 und 2 verschmelzen zu einer einheitlichen Platte mit 69/31. Das Genitale 3 misst 144/56, das Exkretale 187/162 und das Post-Exkretale 243/231.

Gnathosoma: Infracapitulum 218/175. 

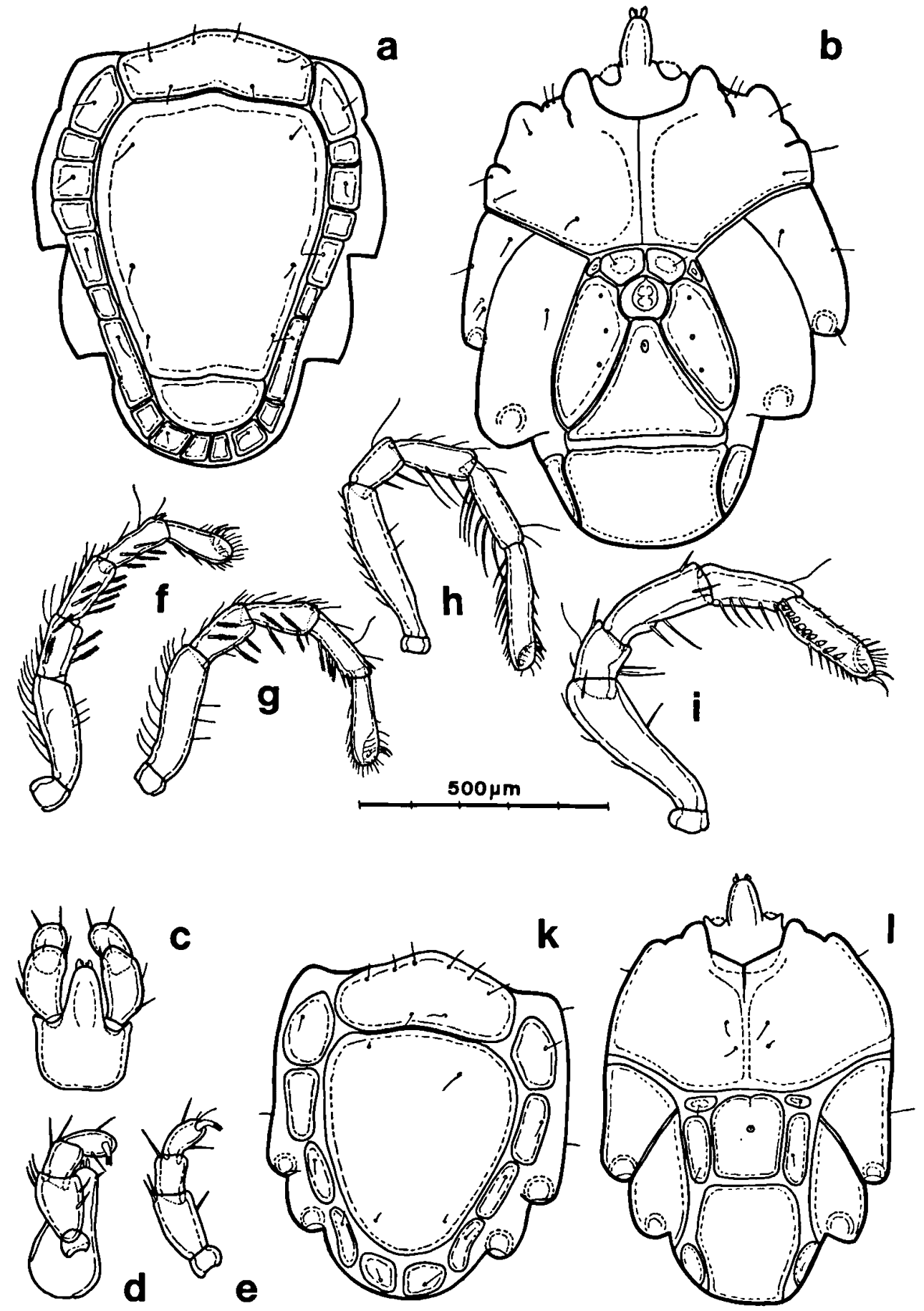

Abb. 3. Neoacherontacarus dividuus nov. gen, nov. spec. Männchen: a, dorsal; b, ventral; c, Gnathosoma dorsal; d, Gnathosoma lateral; e, Palpe innen; $f-i$, Beine I-IV. Nymphe: $k$, dorsal; $l$, ventral. 
Extremitäten:

$\begin{array}{lllrlllll}\text { I } & 62 & 192 & 109 & 109 & 130 & 158 & = & 760 \\ \text { II } & 68 & 201 & 105 & 109 & 139 & 173 & = & 795 \\ \text { III } & 68 & 195 & 96 & 109 & 139 & 176 & = & 783 \\ \text { IV } & 56 & 193 & 93 & 114 & 148 & 179 & = & 783\end{array}$

Die vier Totallängen sind auffallend gleichwertig, wiederum ist das längste Beinglied in der Reihe der zweiten Glieder zu entdecken. Die übrigen Glieder einer Reihe sind nahezu gleich lang.

\section{Panisus clypeolatus Maglio, 1909}

Fundorte: Sta. 83-27 (17.4.1983), Wilaya Tlemcen, Karstquelle, $1 \mathrm{~km}$ nördlich von Aï-Fezza, $34^{\circ} 53^{\prime} \mathrm{N} 01^{\circ} 14^{\prime} \mathrm{W}$, Höhe: $900 \mathrm{~m}$. Temperatur: $17,3^{\circ} \mathrm{C}$. 1 Männchen.

Sta. 83-39 (19.4.1983), Wilaya Tlemcen, Karstquelle am Nordufer von Oued Khemis bei Khemis, $34^{\circ} 39^{\prime} \mathrm{N} 02^{\circ} 7^{\prime} \mathrm{W}$, Höhe: $900 \mathrm{~m}$. Temperatur: $16,0^{\circ} \mathrm{C} .1$ Männchen.

Maglio (1909) beschrieb diese Art aus dem Trentino (Riva) und machte auf die Fusion der beiden Dorsalschilder $\mathrm{dc}_{2}$ aufmerksam. Einige Zeit vorher hatte Piersig (1896) seine $P$. torrenticolus mit der gleichen Eigenschaft eingeführt. Motaş (1928) trennte diese beiden Arten, indem er bei torrenticolus bei den $\mathrm{dc}_{2}$ keine 'komplette Fusion' festzustellen vermochte. Lundblad (1956) verwies auf 'eine nicht unbedeutende Variabilität von torrenticolus', unterschied diese jedoch ohne triftige Gründe von clypeolatus. In meiner Panisus-Publikation (Bader, 1981) wurden die hier angedeuteten Schwierigkeiten zusammengefasst. Die PanisusTiere mit der 'dreifachen Plattenstruktur' gehören zu clypeolatus und torrenticolus, die letztere Art wurde als Sammelbegriff bezeichnet. Die gegenwärtige Situation ist verwirrend! Es scheint, als ob die kaltstenotherme torrenticolus in Nordeuropa (bis zu den Alpen) vorkommt, die eher eurytherme clypeolatus dagegen im Mittelmeergebiet (bis $\mathrm{zu}$ den Alpen). Das mir zur Verfügung stehende Material beweist die von Lundblad erwähnte Variabilität der Dorsalia $\mathrm{dc}_{2}$ und $\mathrm{dc}_{3}$, in ihnen ist kein arttypisches Merkmal zu finden! Möglicherweise könnten in den Ventralschildern entscheidende Differenzen gefunden werden: Populationsuntersuchungen der beiden nicht allzu häufig auftretenden Arten drängen sich somit auf. Die beiden Funde in Algerien bestätigen zunächst das von
Viets (1951) erstmals gemeldete Vorkommen von clypeolatus in Algerien (Rhouffi). Die von Lundblad gegebenen Funde aus Südfrankreich dürften dieser Art zugewiesen werden. Walter (1922) erwähnte sie mit Funden aus den Karawanken und der Südschweiz (Lugano). Meine eigenen Funde stammen ebenfalls aus der Südschweiz (Arcegno/ Locarno). Die beiden zur Zeit in Italien wirkenden Spezialisten A. Di Sabatino (Abruzzen) und R. Gerecke (Sizilien) entdeckten $P$. clypeolatus in ihren Spezialgebieten (mündliche Mitteilungen).

Hydryphantes (s. str.) algeriensis Walter, 1925

Fundort: Sta. 81-4 (7.4.1981), Wilaya Saïda, Sodbrunnen bei Bir-Sénia, $40 \mathrm{~km}$ Nordost von Mecheria, $33^{\circ} 50^{\prime} \mathrm{N} 00^{\circ} 1^{\prime} \mathrm{W}$, Höhe: 1150 m. Tiefe: $22+4$ m. 1 Männchen.

Walter (1925) beschrieb diese Art aus Ost-Algerien: Kabylien, Sumpf zwischen den Seen Oubeira und Tonga. Keine weitere Fundmeldungen.

Nilotonia (Dartia) robusta Walter, 1931

Fundort: Sta. 83-76 (28.4.1983). Wilaya Saïda, gefasste Quelle, $4 \mathrm{~km}$ östlich von Saïda, $34^{\circ} 49^{\prime} \mathrm{N} 00^{\circ} 11^{\prime} \mathrm{O}$, Höhe: $1000 \mathrm{~m}$. Temperatur: $14,2^{\circ} \mathrm{C} .1$ Weibchen.

Walter (1931) fand diese Art in der Central Sahara in einem Sumpf bei Tin-Tahart. Seither wurde sie durch Lundblad (1952) in Ostafrika und Bader aus dem Kongo nachgewiesen. Der neueste Fundort stammt aus Israel, Viets (1981) meldete sie aus einem temporären Teich und aus einem Strom.

Limnesia (s. str.) granulosa Walter, 1931 (Abb. 4)

Fundorte: Sta. $81-4$ (7.4.1981), Wilaya Saida, Sodbrunnen bei Bir-Sénia, $40 \mathrm{~km}$ Nord-Ost von Mecheria $33^{\circ} 50^{\prime} \mathrm{N} 00^{\circ} 1^{\prime} \mathrm{W}$, Höhe: $1150 \mathrm{~m}$. Tiefe: $22+4 \mathrm{~m}$. 6 Weibchen, 7 Männchen.

Sta. 81-21 (16.4.1983), Wilaya Tlemcen, Sodbrunnen, $20 \mathrm{~km}$ Nord-Ost von Sebdou, $34^{\circ} 43^{\prime} \mathrm{N} 01^{\circ} 12^{\prime} \mathrm{W}$, Höhe: $1200 \mathrm{~m}$. Dimension: 1 bei $1,5 \mathrm{~m}$, Tiefe: $2,5+1,5 \mathrm{~m}$, Temperatur: $12,5^{\circ} \mathrm{C}$. 3 Weibchen, 1 Männchen.

Sta. 83-32 (18.4.1983), Wilaya Tlemcen, KaramanChappuis-Grabung am Strand von Sidna Youcha, 7 km OstNordost von Ghazaouet, $35^{\circ} 7^{\prime} \mathrm{N} 01^{\circ} 46^{\prime} \mathrm{W}$, Temperatur: $18,2^{\circ} \mathrm{C}$. 1 Weibchen.

Sta. 83-84 (30.4.1983), Wilaya Tlemcen, Sodbrunnen, $8 \mathrm{~km}$ Süd von Sebdou, $34^{\circ} 36^{\prime} \mathrm{N} 01^{\circ} 20^{\prime} \mathrm{W}$, Höhe: $1100 \mathrm{~m}$. Diam.: 


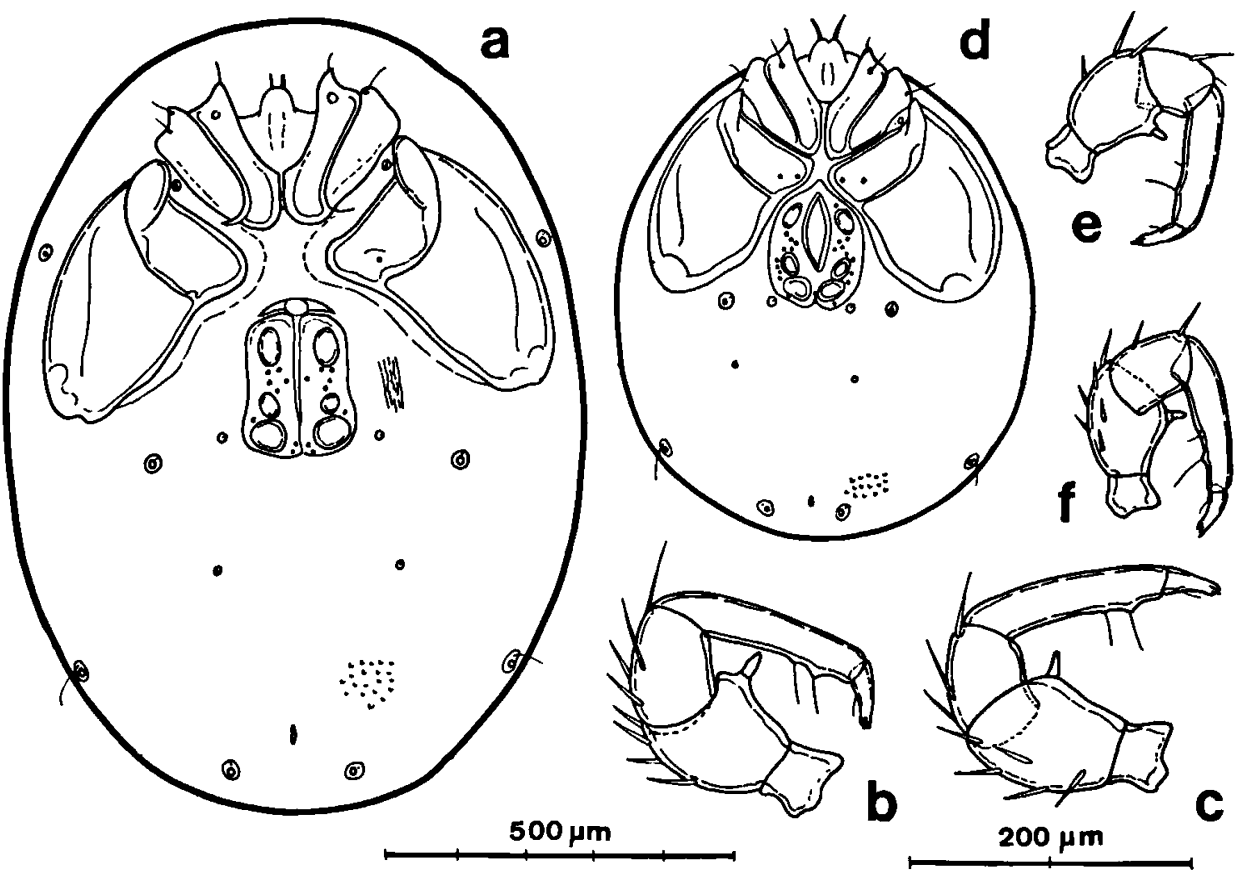

Abb. 4. Limnesia granulosa Walter, 1931. Weibchen: a, ventral; b, Palpe innen; c, Palpe aussen. Männchen: d, ventral; e, Palpe innen f, Palpe aussen.

$1.5 \mathrm{~m}$, Tiefe: $3,5+2 \mathrm{~m}$, Temperatur: $14,2^{\circ} \mathrm{C} .1$ Weibchen.

Sta. 83-96 (2.5.1983), Wilaya Saïda, Quelle, $6 \mathrm{~km}$ West von Tirkount, $32^{\circ} 49^{\prime} \mathrm{N} 00^{\circ} 40^{\prime} \mathrm{W}$, Höhe: $1200 \mathrm{~m}$. Temperatur: $18,0^{\circ} \mathrm{C}$. 2 Männchen, 6 Weibchen.

Sta. 83-97 (2.5.1983), Wilaya Saïda, Sodbrunnen bei BenIkhou, $00^{\circ} 51^{\prime} \mathrm{W} 32^{\circ} 36^{\prime} \mathrm{N}$, Höhe: $1200 \mathrm{~m}$. Diam.: $1 \mathrm{~m}$, Tiefe: $2,5+1 \mathrm{~m} .2$ Weibchen.

Sta. 83-103 (3.5.1983). Wilaya Saïda, Sodbrunnen, $5 \mathrm{~km}$ Nord-Ost von Hadjerat-M'Guil, $32^{\circ} 17^{\prime} \mathrm{N} 00^{\circ} 54^{\prime} \mathrm{W}$, Höhe: $1100 \mathrm{~m}$. Diam.: 1,5 m, Tiefe: $4+2 \mathrm{~m}$, Temperatur: $19,0^{\circ} \mathrm{C} .1$ Weibchen.

Von dieser Art beschrieb Walter (1931) nur das Weibchen, das ihm mit 3 Exemplaren aus der 'Sahara Central' zur Verfügung stand. In seiner Fig. 4 werden Ventralansicht und Palpen dargestellt, die erstere hat sich als mangelhaft erwiesen. Die Kontrolle des im Basler Museum aufbewahrten Typenpräparats XXV/86-89 hat ergeben, dass Walter die auffallende Ausweitung an der dritten Epimere nicht beachtet hat: am äusseren Rand dieser Epimere zeigt sich ein schmaler, nach vorne weisender Wulst. Dieser ist in unseren Abb. 4a und d gut erkennbar. Sonst ist die Walter'sche Diagnose äusserst exakt, die gegebenen Einzelheiten stimmen mit denen unserer Tiere bestens überein, so dass wir uns mit einigen wenigen Ergänzungen begnügen können.

\section{Weibchen}

Ventrum: $890 / 770$ bis $1140 / 940$. Typus des ovigeren Tieres: (1200/930). Eigrösse 175 (165). Epimeralfeld 470/680. Genitalfeld 220/175 (255/180), Papillen 49-31-53. Die von Walter erkannte granulöse Hautstruktur ist nur im hinteren Körperteil ausgebildet.

Gnathosoma: Infracapitulum 160/100. Palpen $31-105-80-155-46=417(35-115-95-170-50$ = 465). Die Angaben über die Behaarung stimmen überein.

\section{Extremitäten:}

$\begin{array}{lrrrrrrrr}\text { I } & 56 & 68 & 96 & 124 & 139 & 142 & = & 625 \\ \text { II } & 56 & 65 & 99 & 142 & 161 & 170 & = & 693 \\ \text { III } & 68 & 87 & 124 & 185 & 204 & 179 & = & 847 \\ \text { IV } & 139 & 124 & 161 & 222 & 222 & 204 & = & 1072\end{array}$

Männchen

Dieses hat sich in 10 Exemplaren gefunden, es 


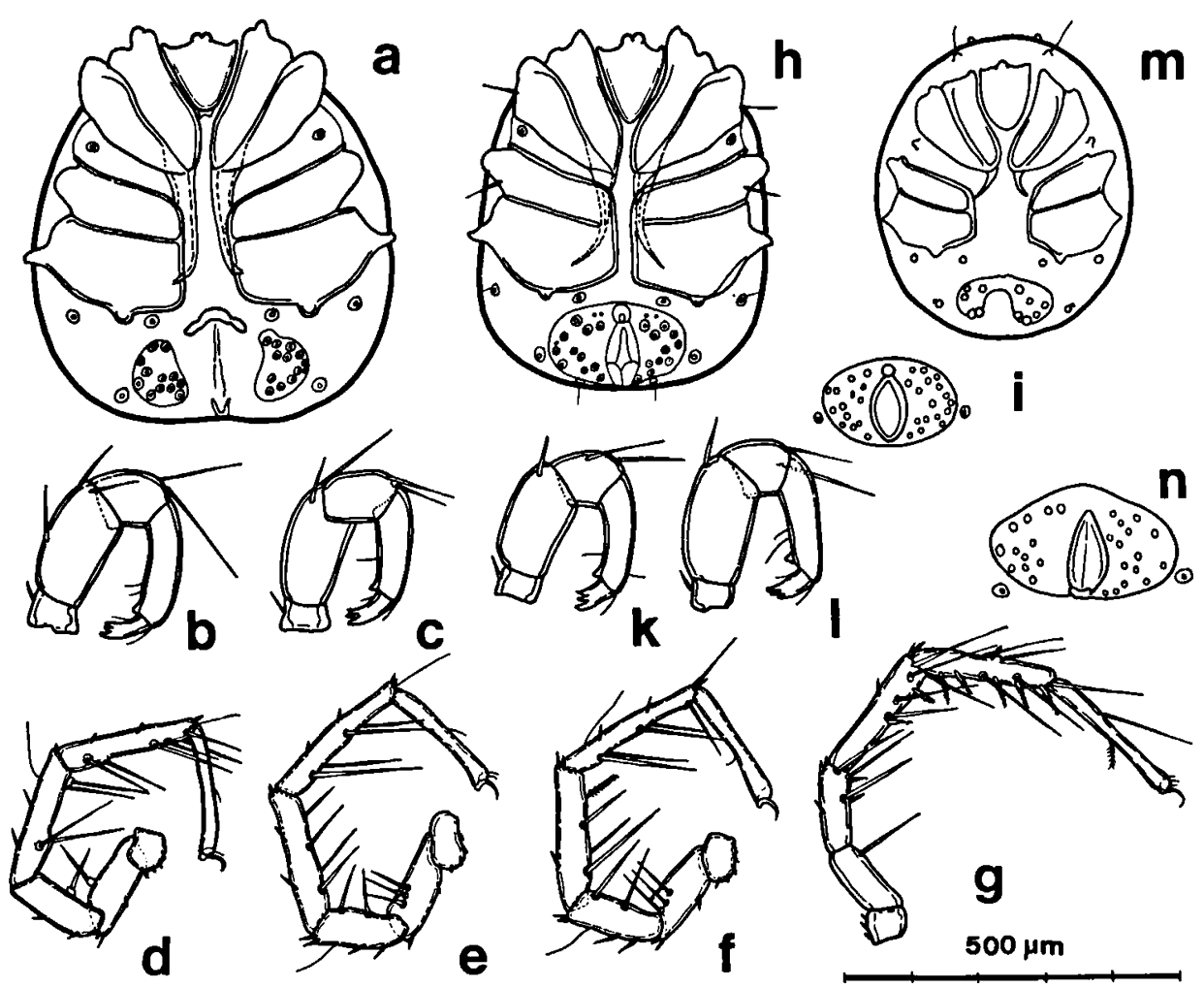

Abb 5. Neumania algeriensis nov. spec. Weibchen: a, ventral; b, Palpe aussen; c, Palpe innen; d-g, Beine I-IV. Männchen: h, ventral; i, Genitalorgan; k, Palpe aussen; l, Palpe innen. Nymphe: m, ventral. Neumania seurati Walter, 1931, Männchen: n, Genitalorgan.

kann neu beschrieben werden.

Ventrum: Körpergrösse 625/585 bis 1070/1070. Epimeralfeld 350/470. Genitalfeld 180/140, Spalte 110, Papillen 43-28-46. Die für die Namensgebungung entscheidende Hautstruktur lässt sich auch beim Männchen nachweisen.

Gnathosoma: Infracapitulum 100/80. Palpen: $19-80-59-114-40=312$.

\section{Extremitäten:}

$\begin{array}{lrrrrrrrr}\text { I } & 49 & 56 & 77 & 105 & 111 & 124 & = & 522 \\ \text { II } & 53 & 62 & 80 & 124 & 139 & 148 & = & 606 \\ \text { III } & 68 & 77 & 90 & 139 & 142 & 155 & = & 671 \\ \text { IV } & 133 & 93 & 117 & 164 & 161 & 155 & = & 823\end{array}$

Neumania (s. str.) elliptica Walter, 1925

Fundort: Sta. 83-111 (4.5.1983), Wilaya Béchar, Sodbrunnen in Oase Menouarar, $31^{\circ} 10^{\prime} \mathrm{N} 02^{\circ} 16^{\prime} \mathrm{W}$, Höhe: ca. $600 \mathrm{~m}$. Diam.: $1,5 \mathrm{~m}$, Tiefe: $4,5+4 \mathrm{~m}$, Temperatur: $22,0^{\circ} \mathrm{C}$. 7 Weibchen.

Walter (1925) beschrieb aus Algerien zunächst nur das Weibchen. Später konnte er (Walter, 1928) in einem weiteren Algerien-Material die Diagnose mit den Beschreibungen der Männchen und Nymphen ergänzen. Viets (1956) verwies in seinem Katalog auf eine Publikation von Margalef (1950), laut der es möglich erscheint, dass die Art auch in Spanien vorkommen könnte.

Neumania (s. str.) algeriensis nov. spec. (Abb. 5)

Fundort. Sta. 83-111 (4.5.1983), Wilaya Béchar, Sodbrunnen in Oase Menouarar $31^{\circ} 10^{\prime} \mathrm{N} 02^{\circ} 16^{\prime} \mathrm{W}$, Höhe: ca. $600 \mathrm{~m}$. Diam.: $1,5 \mathrm{~m}$, Tiefe: $4,5+4 \mathrm{~m}$, Temperatur: $22,0^{\circ} \mathrm{C}$. 25 Weibchen (1 Holotypus), 36 Männchen, 2 Nymphen.

Aus der zentralen Sahara beschrieb Walter (1931) seine Neumania seurati. Unsere hier beschriebene neue Art ist mit seurati sehr nahe verwandt. Sie unterscheidet sich vor allem im Verlauf der von den 1. Epimeren ausgehenden Epidesmen. Bei seurati enden diese am hinteren Rand der 3. Epimeren, bei $a l$ geriensis hingegen erstrecken sie sich weit ins Ge- 
biet der 4. Epimeren. Des weiteren unterscheiden sich die von Walter gegebenen Masse wesentlich von den unsrigen. Leider gestattete der schlechte Zustand unseres Materials (Alkohol-Konservierung!) keine einwandfreie Präparation resp. Beobachtung. Die von uns hergestellten mikroskopischen Präparate besitzen grosse Mängel.

\section{Männchen}

Ventrum: Körpergrösse des Holotypus 500/390 (seurati 645/585), weitere Exemplare zwischen 450/360 und 560/440. Walters Art ist rundlicher. Genitalorgan 206/125 (290/220). Die Zahl der Papillen ist mit 17 resp. 14 etwas grösser als bei der Vergleichsart (14 resp. 11).

Gnathosoma: Infracapitulum 93/99 (160/125). Palpen 28-90-59-102-34 = $313(30-115-60-$ $115-45=365$ ). In der Behaarung zeigen sich gegenüber der Vergleichsart keine wesentlichen Unterschiede.

\begin{tabular}{llrllllll}
\multicolumn{8}{l}{ Extremitäten: } \\
I & 46 & 96 & 109 & 173 & 198 & 188 & $=810(960)$ \\
II & 46 & 102 & 114 & 182 & 216 & 204 & $=$ & $864(960)$ \\
III & 62 & 93 & 102 & 158 & 192 & 222 & $=$ & $829(990)$ \\
IV & 74 & 102 & 114 & 182 & 204 & 204 & $=$ & $980(1065)$
\end{tabular}

Die drei ersten Beine weisen ungefähr die gleiche Länge auf, das vierte Bein ist deutlich grösser. Es fällt daran auf, dass im Vergleich mit vielen anderen Familien der 'echten Hydrachnellen' Neumania als Vertreter der Unionicoliden am Hinterbein eine Sonderstellung einnimmt: das erste Glied vom Hinterbein ist kürzer als die folgenden Beinglieder. Die Behaarung der Vergleichsart konnte nicht untersucht werden.

\section{Weibchen}

Ventrum: Körpergrösse 625/560 (930/750), weitere Exemplare zwischen $610 / 500$ und $1050 / 800$. Genitalorgan 280/190 (400/255). Spalt 170 (215). Papillen 12 resp. 13 (13).

Gnathosoma: Infracapitulum 137/125. Palpen $25-93-56-93-37=304(40-125-70-130-55$ $=420$ ).

\section{Extremitäten:}

$\begin{array}{lrlllllll}\text { I } & 53 & 127 & 155 & 222 & 232 & 210 & = & 999(1005) \\ \text { II } & 53 & 123 & 155 & 235 & 232 & 201 & = & 999(1011) \\ \text { III } & 56 & 102 & 124 & 201 & 232 & 201 & = & 916(1064) \\ \text { IV } & 111 & 127 & 158 & 222 & 266 & 232 & = & 1116(980)\end{array}$

Es konnten nur die Beine der Weibchen gezeichnet werden, über deren Behaarung geben die Abb. 5 $\mathrm{d}-\mathrm{g}$ Auskunft.

Nymphe

Ventrum: Körpergrösse 470/390 (480/390). Die Epidesmen sind sehr kurz. Genitalorgan 140/65 $(145 / 85)$.

\section{Literatur}

Bader, C. 1981. Panisus-Studien: 1. Zur Revision der Gattung Panisus Koenike, 1896 (Acari, Actinedida, Hydrachnellae). Entomol. Bas. 6: 52-77.

Bader, C. 1983. Zwei neue Wassermilben - Arten aus dem Interstitial des Mittelmeergebietes. Rev. Idrobiol. 22: 169-177.

Cook, D. 1967. Water mites from India. Amer. Entomol. Inst. 9: 1-411.

Lundblad, O. 1952. Hydracarinen von den ostafrikanischen Gebirgen. Ark. Zool. 31(3): 391-525.

Lundblad, O. 1956. Zur Kenntnis süd- und mitteleuropäischer Hydrachnellen. Ark. Zool. 10: 1-306.

Lundblad, O. 1962. Wassermilben von den Kanarischen Inseln. Ark. Zool. 15/16: 285-300.

Maglio, C. 1909. Idracarini del Trentino. Atti Soc. Ital. Sc. Nat. 48: 251-296.

Margalef, R. 1950. Datos para la hidrobiologia de la Cordillera cantabrica. Publ. Inst. Biol. apl. Barcelona 7: 37-76.

Motaş, C. 1928. Contribution à la connaissance des Hydracariens français particulièrement du Sud-Est de la France. Trav. Lab. Hydrobiol. Piscic. Univ. Grenoble 20: 1-373.

Notenboom, J. \& A.J. de Winter, 1986. Dutch biological and speleological exploration in Algeria, 5. Survey of the groundwater-stations investigated in 1981 and 1983: 1-120 (Biospeleologische Werkgroep van Speleo-Nederland, Amsterdam).

Piersig, R. 1896-1899. Deutschlands Hydrachniden. Zoologica 19: $1-601$.

Viets, K. 1951. Hydrachnellae (Acari) aus den französischen Alpen und Afrika. Zool. Anz. 147: 287-303.

Viets, K. 1956. Die Milben des Süsswassers und des Meeres: 1-870 (VEB Gustav Fischer Verlag, Jena).

Viets, K.O. 1981. On watermites (Acari: Hydrachnellae) from Israel: Nilotonia robusta (Walter) and a new species of Torrenticola. Israel J. Zool. 30: 22-29.

Walter, C. 1922. Die Hydracarinen der Alpengewässer. Denkschr. Schweiz. Naturf. Ges. 58: 60-251.

Walter, C. 1925. Hydracariens de l'Algérie et de la Tunisie (Collections de M.H. Gauthier). Première liste. Bull. Soc. Hist. nat. Afrique Nord 16: 189-238.

Walter, C. 1928. Hydracariens de l'Algérie et de la Tunisie (Collections de M.H. Gauthier). Deuxième liste. Bull. Soc. Hist. Nat. Afrique Nord 19: 280-336.

Walter, C. 1931. Hydracariens du Sahara central. Bull. Soc. Hist. Nat. Afrique Nord 22: 331-349.

Eingegangen am 3. Juni 1988 